\title{
Connecting Brains and Bodies: Applying Physiological Computing to Support Social Interaction
}

\author{
Guillaume Chanel $^{1, *}$ and Christian MüHL ${ }^{2}$ \\ ${ }^{1}$ Swiss Center for Affective Sciences, University of Geneva, Campus Biotech, 9, Chemin des Mines, \\ Case postale 60, CH-1202 Geneva, Switzerland \\ ${ }^{2}$ German Aerospace Center, Aerospace Medicine/Flight physiology, Linder Höhe, 51147 Cologne, Germany \\ *Corresponding author: guillaume.chanel@unige.ch
}

\begin{abstract}
Physiological and affective computing propose methods to improve human-machine interactions by adapting machines to the users' states. Recently, social signal processing (SSP) has proposed to apply similar methods to human-human interactions with the hope of better understanding and modeling social interactions. Most of the social signals employed are facial expressions, body movements and speech, but studies using physiological signals remain scarce. In this paper, we motivate the use of physiological signals in the context of social interactions. Specifically, we review studies which have investigated the relationship between various physiological indices and social interactions. We then propose two main directions to apply physiological SSP: using physiological signals of individual users as new social cues displayed in the group and using inter-user physiology to measure properties of the interactions such as conflict and social presence. We conclude that physiological measures have the potential to enhance social interactions and to connect people.
\end{abstract}

\section{RESEARCH HIGHLIGHTS}

- The work on physiological computing, affective computing and social signal processing is briefly reviewed.

- Physiological indices of social interactions are reviewed in the context of social psychology and neuroscience and examples of applications are given.

- Two research directions are proposed to use physiological signals for social signal processing:

$\circ$ the display of new physiological and social cues;

$\circ$ the use of multi-person physiological activity as a measure of social interactions.

Keywords: Collaborative interaction; Collaborative and social computing; Human-centered computing; Law social en behavioral sciences; Affective computing; Physiological computing

Received 14 July 2014; Revised 16 March 2015; Accepted 25 March 2015

\section{INTRODUCTION}

Human-computer interaction (HCI) aims at the design of human-computer interfaces that are functional, usable and that induce positive user experiences. Reaching those goals, particularly user experience enhancement, can be achieved by analyzing and properly reacting to users' emotional states as was proposed by Picard (1997) who initiated the field of affective computing. Affective computing aims at the development of machines that are able to recognize and display emotions in order to bring HCI closer to human communication. Emotion recognition can be achieved by several means, for instance through the inference of facial expressions and vocal prosody (Gunes et al., 2008; Zeng et al., 2009). Physiological computing (Fairclough, 2009) is a powerful method to infer information about computer users' states through the collection of their physiological signals 
and has often been used to perform emotion recognition (Chanel et al., 2009; Fairclough, 2009; Lisetti and Nasoz, 2004; Mühl et al., 2014a). Results are now clearly converging, demonstrating the interest and efficiency of this type of signals for affective computing, especially when combined with other modalities (Koelstra and Patras, 2013; Koelstra et al., 2012; Soleymani et al., 2012).

Emotion assessment technology allows for the development of interfaces able to better adjust to human communication. However, emotions are not the only non-verbal signals that humans communicate. There is a wide range of social signals and behaviors (Mehu and Scherer, 2012; Vinciarelli et al., 2012) that should be taken into account by computers to improve HCI. Examples are head nodding, gestures with communicative intention (e.g. signs and emblems) and speaker interruptions. The analysis of those signals and their inclusion in human-machine interfaces has been named social signal processing (SSP) (Pentland, 2007; Vinciarelli et al., 2012). Interestingly, the application of SSP is not limited to the improvement of machines as it can be used to better understand the social interactions taking place in a group of individuals (Pentland, 2006). Analyzing social interactions is particularly relevant with the increasing development of on-line social platforms, collaborative computer supported work and any other form of computer-mediated communication. In these contexts, the computer mediation allows one to enrich the interaction, when compared with face to face interactions, with new social cues (e.g. smileys, age detectors) and to adapt the interaction with the hope to improve it (e.g. providing advice to resolve a conflict).

Research in social psychology and neuroscience has shown that physiological activity is related to several social processes such as empathy (Levenson and Ruef, 1992) and many other social behaviors (Adolphs, 2003; Levenson and Gottman, 1983). In spite of these results, physiological signals have rarely been employed to analyze any social cues other than affective expressions. Given the lack of studies in physiological computing which tries to account for social interactions, the objective of this article is to propose paths to strengthen this innovative research direction. First, a general background on affective computing, physiological emotion assessment and SSP is given in Section 2. Then, Section 3 discusses findings in social neuroscience and physiology which motivate the use of physiological signals for SSP. In Section 4, two directions are proposed to include physiological computing in the field of SSP: the use of physiological signals as new social cues and the use of physiological coupling to measure the nature of social interactions. Potential applications to illustrate those two research directions are given and Section 4 concludes on the current challenges of the domain.

\section{BACKGROUND}

\subsection{Physiological affective computing}

The goal of affective computing is to build machines able to detect non-verbal cues given by users and react accordingly. By including emotions in the human-computer loop (Fig. 1), it is expected that computers will better understand and respond to the needs of their users. A first step toward the design of these intelligent interfaces is emotion assessment. The detected emotional state is then used to synthesize appropriate responses using emotionally expressive agents and to adapt the machine behavior. Affective computing has gained increasing popularity in the last 20 years and is now approaching maturity as reflected by the number of emerging applications. For instance, video games that adjust their difficulty to the player's emotions (Chanel et al., 2012a; Liu et al., 2009) or that can be controlled by self-induced affect (Mühl et al., 2010) have been developed. The maturity of affective computing is also demonstrated by the performance of emotion recognition algorithms which are, in specific situations, able to detect

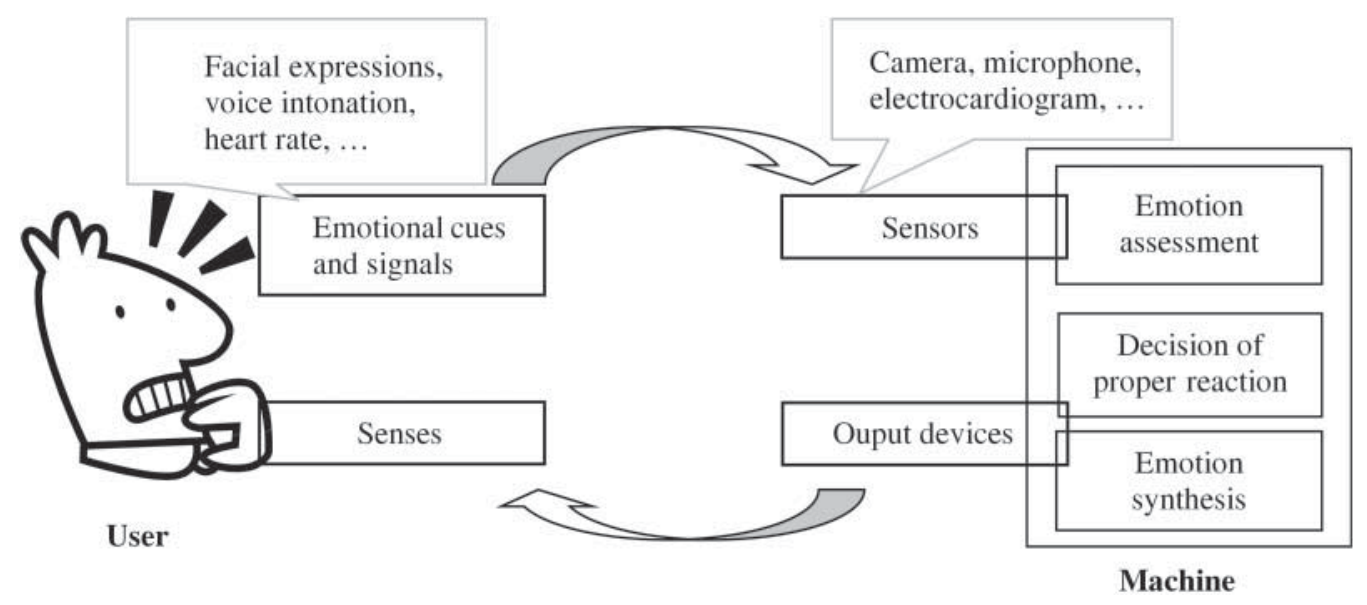

Figure 1. Affective computing aims at including emotions in the human-computer loop. 
emotions (Janssen et al., 2013b) and deception (Bartlett et al., 2014) better than humans.

During human-human interactions, emotions are communicated either verbally or through non-verbal signals such as facial expressions and tone of voice. The video capture of facial expressions together with voice capture are certainly the most studied modalities for the purpose of emotion assessment (Calvo and D'Mello, 2010; Cowie et al., 2001; Gunes et al., 2008; Pantic and Rothkrantz, 2003; Zeng et al., 2009). Bodily emotional expressions are also exploited for this purpose and include the study of body postures (Kleinsmith et al., 2011) as well as movements and gestures (Castellano et al., 2007, 2010; Gunes et al., 2013). Finally, given the importance of physiology and cognition in emotions (Ortony et al., 2007; Sander et al., 2005), physiological signals, reflecting the activity of the central and peripheral nervous system, have also been employed for emotion assessment (Calvo and D'Mello, 2010; Chanel et al., 2009; Fairclough, 2009; Mühl et al., 2014a).

When compared with other sources of emotional information, such as speech, gestures and facial expressions, physiological signals present several advantages for emotion and experience assessment (Kivikangas et al., 2011; Mandryk et al., 2006; Mühl et al., 2014a): they are mostly involuntary and as such are quite insensitive to deception, they can be used to measure the affective states continuously, and physiological reactions can be observed as soon as $200 \mathrm{~ms}$ after emotional stimuli in the case of neurophysiological signals. In addition, physiological signals are not part of the modalities used by humans to recognize others' emotions which imply that they could be used to augment human-human affective communication. Finally, in the case of impaired users that cannot move facial muscles or express themselves, several physiological signals, for example neurophysiological activity, are still available for emotion assessment. All these arguments motivate the use of physiological signals in conjunction with other affective signals.

\subsection{Social signal processing}

Among emotion theories, the social constructivist view (Cornelius, 1996) has emphasized the role of the social context in shaping emotions as well as the importance of emotions for social interactions. On the one hand, the role of the social context is illustrated by the results of Jakobs et al. (1997), showing that co-experience and co-expression of an emotion increases the intensity of the felt emotion. Social phenomena such as emotion contagion, empathy (Hatfield et al., 2009) and conflicts also demonstrate how the emotional climate in a group can influence one's emotions. On the other hand, emotions have important functions in social situations. According to Van Kleef (2009) emotional expressions provide information about the producer which are then used by the observers to adjust their social behavior. For instance, it has been shown (Van Kleef, 2006) that displayed emotions can strongly influence the result of a negotiation, particularly in the case where a difference of power exists between negotiators. It is thus clear that social contexts (e.g. relationship or social situations such as negotiation) and emotions influences each other's, although the exact processes taking place are still under discussion.

Considering the importance of social skills in human interactions, a new field of research has recently emerged; named SSP (Pentland, 2007; Vinciarelli et al., 2012). It 'aims at providing computers with the ability to sense and understand human social signals' (Vinciarelli et al., 2009). SSP is thus very close to affective computing in the sense that it tries to create machines able to better understand and respond to humans. However, SSP goes beyond affective computing in several aspects. First, it generally considers signals that are exchanged in a group and thus is based on the analysis of several entities, whether human or machines, while affective computing often concentrates on a unique human-machine interaction. The group setup allows for the study of behaviors, affective expressions and psychological constructs both at the level of the individual and at the collective level. For instance, intelligence can be regarded as an individual property. However, when several people combine their knowledge the result is often more than the sum of individual knowledge, giving rise to collective intelligence (Pentland, 2006). Similarly, the collection of individual affective exchanges between the members of a group can be used to define intersubject concepts such as group mood (Lehmann-Willenbrock et al., 2011). Secondly, the range of signals, cues and behaviors considered are wider than in affective computing. While affective computing studies emotional states, SSP also encompasses phenomena such as disagreement, ambivalence, and attention. Where affective computing analyses expressions such as facial expressions and prosody, SSP also studies signals such as emblems and manipulators (Vinciarelli et al., 2009).

The unit of analysis of SSP is the social signal, it is thus worth defining what a social signal is. Generally speaking, a signal communicates information from a sender to a receiver. In behavioral sciences, social signals can be seen as structures or acts that have evolved to influence the state and behavior of the receiver to the advantage of the sender (Mehu and Scherer, 2012 ). For instance, a facial expression (e.g. displaying sadness) could be considered as a social signal since its perception by others changes their behaviors, generally to profit the sender (e.g. compassionate behaviors). In Mehu and Scherer (2012), the authors mention the difference between cues and signals. A cue gives information on a quality of the sender although it was not primary selected for that purpose. The difference between a cue and a signal is subtle and it is often difficult to distinguish them. However, the difference remains important as cues are expected to be more reliable than signals since they are less prone to deception. In this framework, the perceivable physiological activity could be 
defined as a cue, since (i) it is primarily function is to support biological maintenance and not to affect others perception, (ii) it can provide information about an individual's inner state. For example, blushing might be considered as a cue since it communicates information about the internal sate of the sender although it is caused by an increase of blood pressure which main function is to support action.

Considering physiological signals as cues implies their measurement could be used as a reliable modality for SSP. So far several sensor modalities and signals have been used to infer social information from interaction, including video and speech analysis. In addition, SSP has also used RFIDs and cell-phones to measure the amount of direct and mediated interactions (Pentland, 2006). A review of the different technics employed in SSP is not the objective of this paper and interested readers can refer to Vinciarelli et al. (2009, 2012). However, we give below a few examples of SSP applications in order to give a better understanding of the field.

Many applications in fields as diverse as marketing, multimedia indexing and retrieval, robotics and HCI can be improved or made possible by SSP (Vinciarelli et al., 2012). A first example of an application of SSP is the detection of the roles taken by group members. In Sanchez-Cortes et al. (2012), the automatic detection of emergent leaders in small groups is achieved based on the analysis of multimodal group features such as turn taking and mutual gazes. This technology could, for instance, be employed to support leaders' recruitment strategies by allowing the detection of natural leaders. Another field of particular interest is computer-mediated communication. When interacting through computers several social signals are lacking due to missing channels (e.g. no web-cam to communicate face movements) or distorted by the communication channels (e.g. low webcam quality). This could have some impact on social interaction processes and on the sense of social presence, the feeling of being in contact physically and psychologically with the other (Biocca and Harms, 2003b; Sallnäs, 2005). In this area, SSP can be useful in several ways. First, it could help to better understand the impact of missing signals on social processes and collaborative outcomes. Understanding the role of these social signals would then be the basis to develop methods for the display of new emotional cues determined from non-visible expression of affect such as physiological signals. Secondly, it could be employed to measure social presence based on users' social signals as proposed in Ekman et al. (2012). Finally, SSP could be used to automatically perform social inferences. In this last scenario, Pesarin et al. (2012) proposed a semi-automatic system to detect moments of conflicts during interactions, while Chanel et al. (2013) proposed to assess several key collaborative processes from the analysis of group physiological signals and eye-movements. In both cases, the inferences could be employed to adapt the mediated interface, for instance by providing help to resolve conflicts.
Using physiological cues for SSP was proposed in Vinciarelli et al. (2009). Despite this encouragement there are to date only a few systems that use physiological signals to perform social inferences (not considering the systems from affective computing dedicated to emotion assessment). As will be detailed in Section 3, human physiology provides the resources necessary to express social signals and cognition which are essential for complex social responses (Uddin et al., 2007). Consequently, we advocate for the use of peripheral and central physiological signals for social inferences.

\section{SOCIAL SIGNALS IN PHYSIOLOGY AND NEUROPHYSIOLOGY}

Physiological measurements have been taken since almost 90 years to study social interactions (see Cacioppo et al., 1989). First, social psychophysiology and later also social neuroscience tried to identify the physiological basis of social constructs, processes and behavior. Today, in the context of SSP, there is a renewed interest in overt and covert physiological signals as valuable social cues. In the face of their potential value, the question is what kind of signals can be used, that is which type of peripheral or central physiological activity carries direct or indirect information about the state of the users and about the quality of the interactions between them? Mehu and Scherer (2012) mention affect, interpersonal attitudes and personality characteristics as concepts relevant to social interaction. Besides directly influencing the social relationship, such states also carry information about the context of the interaction, the internal environment of signaler or perceiver. They could, therefore, be important cues for SSP systems. Moreover, the quality of inferences depends on the quantity and quality of available data: additional signal sources (e.g. neurophysiological measures) would add to both by delivering complementary and redundant information about user state. For example, the communicative intention of a certain social signal might be ambiguous and therefore needs contextual information about social behaviors, affective, cognitive states (Vinciarelli et al., 2009).

Therefore, we can identify several lines of research in psychophysiology and the neurosciences that are relevant to SSP, namely those underlying the affective, cognitive and social sciences. Below, we will briefly describe the insights and potential contributions of these different research domains that are relevant for SSP.

\subsection{From individual affective and cognitive signals to social signals}

For decades, psychophysiologists and neuroscientists have studied the physiological and neurophysiological correlates of affect and cognition. As mentioned before, affective states can be relevant social cues and their observation can lead to 
the adaptation of behavior in social interactions. Knowing, for example, the emotional response of a person to an event or to an interaction with another agent (i.e. a person or a machine) can be informative to predict the reactions of this person in future interactions and consequently how to behave in order to avoid undesired reactions (e.g. anger, anxiety, etc.). Similarly, the cognitive states of interlocutors can be used to determine the most interesting conversational topics for the group.

\subsubsection{Affective signals}

Different signals assessing the peripheral and central nervous system activity have been associated with emotional responses (Kreibig, 2010) and found relevant for the detection of emotions (Mühl et al., 2014a; Novak et al., 2012).

For the peripheral nervous system, two of the most informative signal varieties are electrodermal activity (EDA) and cardiovascular activity. EDA is related to the changes of electrical potentials and resistance of the skin (Féré, 1888) which varies with perspiration. EDA can be used as a direct measure of physiological arousal and has been shown to be correlated with self-reported arousal (Lang et al., 1993). Significant differences in EDA have also been observed between pleasant and unpleasant stimuli (Delplanque et al., 2008). Cardiovascular activity, such as heart rate or its variability, can be measured in the form of electrical potentials from the heart (electrocardiograms-ECG), heart sounds and the changes of relative blood pressure over time, known as blood volume pulse. Increase or decrease of HR properties can be associated with different emotions (Ekman et al., 1983; Rainville et al., 2006). Many other physiological signals have been used to detect emotions, including facial EMG signals to detect expressions of affect, respiration and skin temperature (Broek et al., 2006; Chanel et al., 2011; Hazlett and Benedek, 2007; Hussain et al., 2011; Katsis et al., 2008; Wilhelm et al., 2006).

For the central nervous system, several neuroanatomical structures are known to be involved in the processing of affective information, such as amygdala and the limbic system, orbitofrontal and medial-prefrontal cortices (see Barrett et al., 2007). Despite the long-standing debate about their specific involvement (with constructivists (Lindquist and Barrett, 2012) versus localist (Vytal and Hamann, 2010) positions as extreme poles), there is a long line of research showing the accessibility of affective states from neurophysiological measurements. Especially for electroencephalography (EEG), measuring the electrical potentials from the brain, several characteristics of brain activity have been found sensitive to emotional stimulation and states. For example, the socalled frontal alpha asymmetry (Coan and Allen, 2004; Schmidt and Trainor, 2001), measuring a lateralization of the brain activity toward either the left or the right frontal cortices, has been found indicative of the valence dimension, pointing to a positive versus negative feeling, respectively. ${ }^{1}$ Another indicator of affect is the overall amount of alpha activity, which decreases with increasing arousal and increases with relaxation (Barry et al., 2007, 2009; Niedermeyer, 2005), though especially posterior and fronto-central alpha oscillations might rather indicate the activity of relevant sensory cortices in response to arousing stimulation than general emotional arousal (Mühl et al., 2011). An extensive overview of sensitive EEG signal characteristics and affect classification approaches can be found elsewhere (see Mühl et al., 2014a).

In a social context, specific emotions can arise which necessitate the evaluation of the other's belief, feelings or actions (Hareli and Parkinson, 2008). These are generally called social or moral emotions and include emotions such as shame, embarrassment, gratitude and admiration (Hareli and Parkinson, 2008; Tangney et al., 2007). Most of the research carried out in the domain of affective computing has focused on basic emotions and dimensional models of emotions. The design of new methods to infer social emotions is thus necessary to develop machines able to react to complex situations such as those encountered in socialmediated interactions.

\subsubsection{Cognitive signals}

Besides affective states, there are also certain cognitive states that can be used for the analysis of group interactions and that can be accessed by physiological and neurophysiological signals (Novak et al., 2012). In the peripheral nervous system, cardiovascular activity, for example (respiratory sinus arrhythmia), has been shown to correlate with attention (Ravaja, 2004). Blood pressure also indicates the motivation and efforts put in a task (Gendolla and Richter, 2005). EDA has been associated with cognitive arousal and thereby with cognitive effort (Boucsein, 1992; Verwey and Veltman, 1984).

Cognitive neuroscience studies a wide spectrum of neurophysiological concepts and processes, such as perception, attention and decision-making, which are also relevant for social interactions and can give insight into social relationships. One example is the determination of the level of attention to others, which might indicate if a person is really paying attention to another person or only looking at the person. Posterior alpha activity, for example, is known to decrease with increasing visual attention (Pfurtscheller et al., 1996) and can be used to infer the attentional focus in a scene (Treder et al., 2011). Such information could help to identify dominant people, those that draw attention, within a group. Also different levels of cognitive load can be distinguished via neurophysiological signals: prefrontal activity in the form of theta oscillations and alpha oscillations (Jensen and Tesche, 2002;

\footnotetext{
${ }^{1}$ Alternatively, researchers have identified a dimension of motivational direction, spanning from approach to withdrawal intention (Davidson, 1995; Harmon-Jones, 2003).
} 
Klimesch, 1996; Laursen et al., 2002; Mühl et al., 2014b), as well as pre-frontal blood flow (Herff et al., 2013; Sassaroli et al., 2008) vary with mental effort and can be used as indicators of mental engagement in group settings.

Summarizing, an individual's affective and cognitive states have robust correlates in peripheral and central nervous system activity. These might be used as indicators of the valence/motivational direction or arousal associated with an encountered (social) event or of the level of (social) attention and engagement in an interactive setting or encounter. In the next section, we will look at more direct indicators of social cues, as studied by social psychophysiology and neuroscience.

\subsection{Multi-person physiological activity as measure of social interactions}

The majority of research in psychophysiology and the neurosciences is done on the single individual. The subfields of these research disciplines that work on social interaction and cognition have, despite their focus on the interactions between individuals, focused on 'passive observers' instead of 'active agents' (Schilbach et al., 2013). However, the processes that can be observed during active interaction-much more characteristic of social processes than a passive participation-might be fundamentally different. Psychophysiological researchers already experimented decades ago with simultaneous measurements of interacting participants.

Social psychophysiologists proposed a method for the analysis of the inter-dependency of behaviors in social interactions already in the early 1980s (Gottman and Ringland, 1981; Allison and Liker, 1982). This measure can be used to quantify the amount of inter-dependency between the behaviors of two people and to identify if the behavior of one person predicts the behavior of the other. Levenson and Gottman (1983) used such a method to measure the physiological coupling of spouses during marital conflicting situations. The coupling index, named physiological linkage, was computed from peripheral physiological signals (heart rate, EDA and general somatic activity), and was found to capture mainly negative social interactions. Interestingly, physiological linkage was also found to be associated with empathy (Levenson and Ruef, 1992); people who accurately evaluated the negative emotions of others also displayed a high degree of shared physiology. This might be due to emotional convergence, an important component of empathy (Janssen, 2012), which is responsible for the alignment of peoples' emotional states through mimicry and imitation. Taken together these studies show that the coupling between peripheral physiological signals of interacting people can be related to several characteristics of the interaction, including empathy, emotional convergence, conflicts and behavioral entrainment.
Social neuroscience-the study of the neural basis and mechanisms of social interaction and $\operatorname{cognition}^{2}$ (Sänger et al., 2011) - until more recently, has relied exclusively on single and passive participant studies. Two systems have been identified that seem at the basis of social interaction and cognition: the mirror neuron system (MNS) and the mentalizing system (MS). Mirror neurons-found in the structures of the motor system, such as the premotor cortex, the supplementary motor area, the primary somatosensory cortex and in the inferior parietal cortex - are active during own and observed actions (Molenberghs et al., 2009; Rizzolatti and Fabbri-Destro, 2008). Moreover, they were associated with the goal-orientation, independently of the effector (e.g. hand), which makes them part of a potential mechanism to represent not only others actions, but also their goals. The MNS is therefore important for internal representation of others' actions, their goals and even their emotions (Rizzolatti and Craighero, 2004). However, the MNS cannot explain causes of actions and emotions (Frith and Frith, 2006) or predict the goals and intentions of new or unexpected actions (Kilner and Frith, 2008). The second network, the 'mentalizing' networkincluding structures as the posterior superior temporal sulcus, temporal parietal junction, the temporal poles and the medial prefrontal cortex - is thought important for inferring the intentions of others in situation where the observed action is novel or difficult to understand (Frith and Frith, 2006). Both networks are supposed to interact to be able to infer and represent others' goals and mental states, though the precise nature of this cooperation is yet unclear (Barrett and Satpute, 2013).

Recently, a developing 'second-person neuroscience' started exploring novel methods for the study of neurophysiological correlates of real-time, active interaction, turning away from traditional single to more realistic multiple-subject studies of social interaction (Schilbach et al., 2013). A number of novel experimental approaches use the 'hyperscanning' paradigm to evaluate the neurophysiology of actual social interaction. Hyperscanning techniques aim at the creation of 'spatiotemporal maps of cerebral regions involved in the generation of the social task investigated' in a study (see Babiloni and Astolfi, 2012). They are based on the recording of the neurophysiological activity of two or more interacting people via functional magnetic resonance imaging, positron emission tomography, functional near-infrared spectroscopy (fNIRS) or EEG, and on the use of advanced correlation methods to infer the flow of information 'between the brains'. Similar to physiological linkage measures, these methods might be able to reveal information about collaborating or competing subjects that independent neurophysiological measurements might be

\footnotetext{
${ }^{2}$ Social interaction is the capability of active, autonomous agents to engage with other agents in dynamic turn-taking behavior according to social rules on the basis of their perceptions of the others' intentions. Social cognition is the capability to understand others, and includes the capability to represent the mental states and intentions of others, known as mentalizing or 'theory of mind'.
} 
blind to. According to Babiloni's and Astolfi's (2012) review of the hyperscanning literature, one can discriminate several experimental approaches that investigate the synchronization of neurophysiological signals from simple dynamical social interaction and communication to social decision-making, and that might be a first step toward reliable social neuromarkers.

Already single-subject EEG studies found evidence for the involvement of the MNS in coordinated social interactions. Tognoli et al. (2007) found that right central-parietal brain activity in the mu band of the $\mathrm{EEG}^{3}$ varied with the presence of spontaneous social interaction. A phi(1) oscillatory component was observed during independent movement, while short bursts of a phi(2) oscillatory component marked coordinated behavior. Though these neuromarkers of social coordination were not present in all subjects, they might be an indicator of suppressed and enhanced activity of MNS, respectively. Studying instructed social interactions, Naeem et al. (2012) found a stronger decrease of power in the right central-parietal mu-rhythm for anti-phasic, compared with phasic, compared with free finger movements, reflecting the integration of mutual information that enables the dynamics of social interaction to unfold over time.

This involvement of putative MNS structures for simple social interaction has been reproduced in multi-subject hyperscanning studies: for intentional as well as for unintentional hand movement coordination (Dumas, 2011; Dumas et al., 2010; Ménoret et al., 2014) and the synchronous playing of musical instruments (Babiloni et al., 2012; Lindenberger et al., 2009; Müller et al., 2013), as well as for more complex interactive scenarios, for example face-to-face communication (Jiang et al., 2012) or aircraft take-off and landing (Astolfi et al., 2011a,b). Besides a symmetrical coupling component, potentially signifying a shared action representation relative to the goal of the action (Ménoret et al., 2014), some studies have found asymmetric components that were related to the role assignment, leader versus follower, during the interaction (Dumas et al., 2010; Konvalinka et al., 2014; Ménoret et al., 2014). Taken together, the studies on temporally coordinated joint action show that hyperscanning paradigms can reveal additional information about the quality of interaction and even leader-follower relationship-potentially based on neurophysiological correlates of the MNS.

The study of social processes beyond simple temporal coordination, for example decisions for or against cooperationinvolving predictions of others' mental states or intentionsrequires more complex experimental approaches. Using interaction paradigms derived from game theory, such as the prisoner's dilemma, as well as traditional games, several studies found an increased connectivity between players' prefrontal cortices, regions supporting mentalizing and decision-making.

\footnotetext{
${ }^{3}$ The mu-rhythm is a frequency band $(8-13 \mathrm{~Hz})$ in the EEG over the sensorimotor cortices, which is closely associated with the perception and coordination of muscle and joint motion.
}

Babiloni et al. (2007a) found a higher activity of the anterior cingulate cortex in the defect condition of the prisoner's dilemma. Furthermore, dorsolateral prefrontal and orbitofrontal activity was higher for defect compared with cooperation condition (Astolfi et al., 2011a,b; De Vico Fallani et al., 2010). Players' central-frontal regions were also found to be more active and connected before decisions in the ultimatum's game (Yun et al., 2008). Using a simple card game, Babiloni et al. (2007b) found higher synchronization of collaborating player's brains compared with competing players, especially for regions involved in decision-making (Astolfi et al., 2010). Finally, Cui et al. (2012) found increased interpersonal coherence in frontal fNIRS signals during cooperation compared with competitive condition of a simple two-player response task.

Summarizing, physiological and neurophysiological measures of inter-subject synchrony have been found informative regarding the social relations and interactions between subjects: as indicators of the strength of social interaction, as indicators of the quality of the interaction (e.g. dominance), and as indicator of intention to collaborate. They potentially can be used to infer such information and thereby provide means to analyze and even aid human-human interaction. However, as for individual indicators of affective and cognitive state, conceptual and methodological limitations have to be identified to ensure reliable assessment of social interactions (see Section 4.4).

\section{APPLYING PHYSIOLOGICAL SSP}

As detailed in the last section, social neuroscience and psychophysiology have put significant effort on the analysis of brain structures and processes which support social interaction and cognition. The results of the research demonstrate that signals from both the central and the peripheral nervous systems carry relevant information on social processes and on personal relationships. Therefore, we advocate the use of physiological signals in SSP and propose two possible solutions to investigate this research direction, one at the individual level and the other at the group level. At the individual level, the measured physiological activity can be used to provide new cues that are not available in current social interactions (Fig. 2). At the group level, modeling the relationships existing between physiological activities of the individuals of a group can be employed to infer properties of the interaction (Fig. 3). The current research achieved in these domains will be reviewed below together with a discussion on potential applications and challenges.

\subsection{Individual physiological social cues}

Physiology plays an important role in the representation and feeling of our own internal state as it constantly gives us 


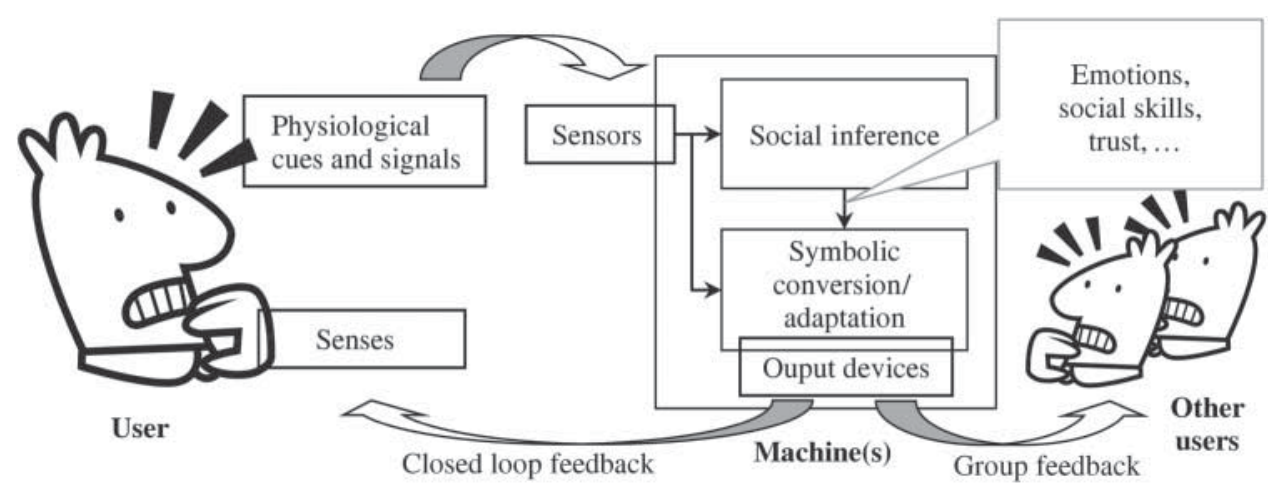

Figure 2. Physiological activity as new cues for social-mediated interactions.

feedback of what is happening in our body (Damasio, 2001; Sander et al., 2005). In addition, some physiological changes can also be perceived by others and thus inform them about our feelings. For instance, a high blood pressure and vasoconstriction can lead to blushing while high physiological arousal can induce shakes, changes of voice acoustic parameters and piloerection. As controlling these physiological cues is very difficult, they are considered as highly reliable by observers and therefore important in social interactions. However, several physiological changes are not perceivable from the mere observation of someone, either because they are too subtle (e.g. heart beat sound) or because they are not displayed to the outside. It follows, that neuro- and physiological signals cannot be directly considered as social signals. However, with the current technology, measuring physiological activity and overtly displaying it becomes feasible. This would thus be a way to provide new social cues and to better inform people about other's internal state (Fig. 2).

The effect of displaying physiological cues remains on open question that only a few studies have investigated, either by using synthetized physiological activity (Janssen et al., 2010, 2013a) or by creating systems able to record and share physiological activity of peers in real time (Chanel et al., 2010; Slovák et al., 2012). Synthetized physiological signals allows creating controlled experiments to study how physiological cues can influence social processes and to better understand which features of physiological cues are more relevant. For instance, the hearing of synthetized heart beats was found to be associated to higher self-reported intimacy and higher interpersonal distance (Janssen et al., 2010). Given that interpersonal distance is known to increase when nonverbal cues are available these results tend to demonstrate that heart beats can be understood as any other non-verbal cues and can help to create intimacy among people. Social heartbeat feedback has been studied in more depth in Janssen et al. (2013a). The authors demonstrated that an increase in other's heart rate is interpreted as an increase in the intensity of their feelings. Interestingly these effects were comparable with those obtained by showing facial expressions and the effect of heart rate remained when facial expressions were also available.

Studying the effect of physiological cues in social situations using the actual physiological activity of the participants, as opposed to synthesized activity, is essential to validate the applicability of physiological cues. In Chanel et al. (2010), the effect of displaying participants' heart rate during the socially mediated watching of movie clips was analyzed. In this experiment, four remote participants were watching a movie together in four conditions: without any feedback, with the possibility to share their experience through chat, with their heart rate continuously fed back to all participants, and with a combination of both chat and physiological feedback. It was demonstrated that displaying the heart rates of participants increased co-presence but only when the chat was not available. This shows that physiological feedback is not only related to emotional aspects but also to the awareness of the other's presence. However, contrarily to the effect on emotional intensity (Janssen et al., 2013a), the effects observed on co-presences seem only significant when other communication channels are not available. The effects of emotion perception and feeling of co-presence were validated by a qualitative study (Slovák et al., 2012) analyzing the users' reactions to a technology which shares heart rate information among peers. This technology was employed in the laboratory (movie watching and negotiation task) and during everyday life. Users reported that they used the technology to infer the emotional state of their partners and that it helped them to feel more connected with their partner when they were physically separated.

The effects of the display of physiological cues are certainly influenced by how the physiology is represented to the users. First, it is of course possible to employ any type of traditional output modality. For instance, the physiological activity can be visualized, with heart beats represented by heart shapes that pounds at the same rhythms that the user's heart beats (Chanel et al., 2010). Another strategy is to transform the physiological signals into sounds (Janssen et al., 2010, 2013a; 


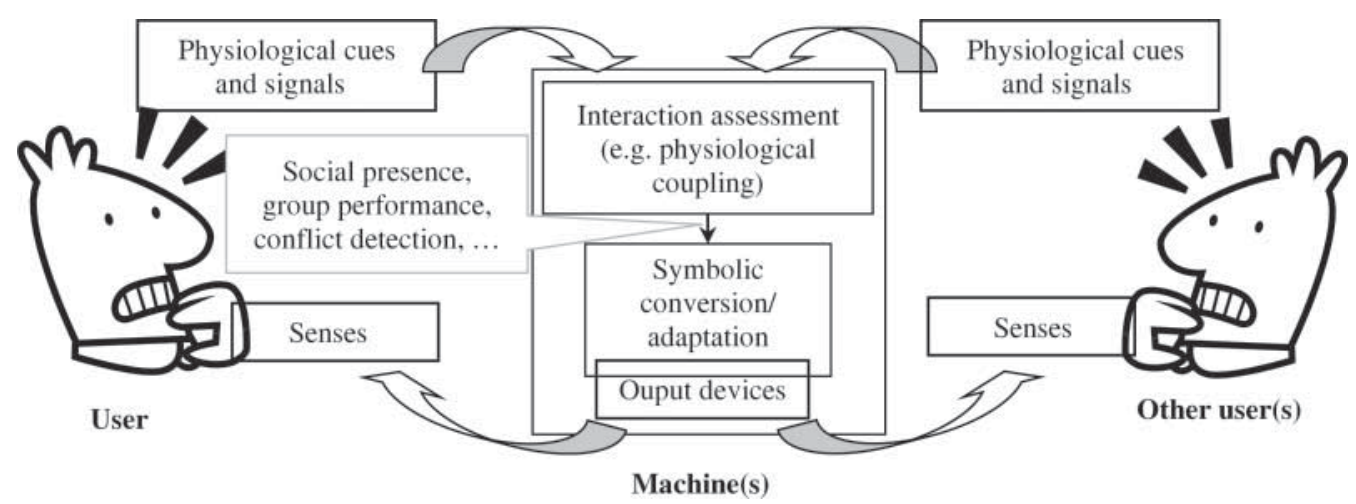

Figure 3. Physiological activity as a measure of social interaction at the group level.

Kosunen et al., 2010). Although not yet investigated, haptic feedback of physiological cues could also be of interest. For any of these modalities the physiological activity needs to be converted into symbols that are understandable by, or at least provide relevant information to the user (e.g. the displayed beating hearts). To our knowledge there are no studies which have investigated the benefits and limitations of the different modalities and symbolic representations for biofeedback. This area thus remains to be explored.

Secondly, instead of displaying physiological activity directly through symbols and representations, it is also possible to provide users with inferences made from the analysis of the recorded signals (Fig. 2). This is what is already achieved in affective computing with the development of methods for users' emotions inference. Section 3 already emphasized affective and cognitive signals which are of interest for SSP.

\subsection{Measuring the interaction from multi-person signals}

The last section argued that social interactions can be studied by analyzing the cues and signals produced by each person independently, or by studying the physiological interdependence/synchrony of interacting individuals. For instance, it is possible to study voice pitch of each partner of a group, but analyzing how the group dialogue develops over time, how speakers interrupt each other's, which speaker is more likely to follow another one, informs about the structure of the group interaction (Delaherche et al., 2012). Similarly, it is possible to analyze physiological signals at the group level, which can be achieved mainly by using physiological coupling, hyper scanning and neural synchrony as detailed in Section 3. Several methods exist to measure the coupling of physiological signals ranging from the computation of the correlation index and coherence analysis to the more complex granger causality criterion and measures of nonlinear dynamic systems synchrony. An extensive review of the existing methods is out of the scope if this paper but the interested readers can refer to the following articles: Delaherche et al.
(2012), Ekman et al. (2012), Jalili et al. (2014), Le Van Quyen et al. (2001).

Although physiological coupling is induced by interactions and has been observed in different social situations, it has rarely been applied to index and predict the quality of these interactions (Fig. 3). An example of such studies is given by Henning et al. (2001) who demonstrated that many of the physiological coupling measures computed from heart rate, EDA and respiration are correlated with task completion time with higher coupling indicating a better performance. The task under consideration was a game where the participants had to synchronize their movements to move a cursor in a maze. However, this positive relationship was not confirmed in a second study (Henning et al., 2009), where self-reported group performance was negatively correlated with physiological coupling.

In Chanel et al. (2012b), the authors suggest that physiological coupling might better index properties of the interaction than the performance on a collaborative task. In this work, the coupling between two players during competitive and collaborative video gaming was studied for several physiological signals (EMG, EDA EMG, heart rate, respiration). The results showed that coupling was higher during competitive play when compared with collaborative play suggesting that it might be a reliable indicator of conflicting interactions. In addition, higher physiological coupling was positively correlated with the sense of social presence (Biocca and Harms, 2003b) which motivates the idea that physiological coupling increases which rich, but not necessarily negative interactions. Social presence, the sense of being together during a mediated interaction, can be decomposed in three components (Biocca and Harms, 2003a,b): copresence which is defined as the sense of being located in the same place, psychological engagement which refers to interpersonal understanding and attention, and behavioral involvement which represent the inter-dependency of behaviors. Since physiological coupling can emerge from both emotional and behavioral synchrony it is able to provide information about at least two of the components of social presence: psychological 
engagement and behavioral involvement. Physiological coupling can thus be regarded as a relevant measure of social interactions which should be more studied for SSP.

Although the two previous examples demonstrate that physiological coupling could be applied to interactive situations such as games, they did not demonstrate the feasibility of prediction employing pattern recognition technics and validation. Peripheral physiological coupling together with eye-movement coupling were employed in Chanel et al. (2013) to predict collaborative processes. In this experiment dyads were collaborating on a task with a common objective and where asked to fill up questionnaires on the interaction. Several continuous scales describing their interaction were obtained from the analysis of the questionnaires and several measures of physiological coupling (ECG, respiration, EDA, temperature) were used to train regression models to assess the scales' values. The crossvalidated results demonstrated that physiological coupling could predict how much participant reported to manage their emotions $\left(R^{2}=0.2\right)$. Emotion management is regarded as an important feature of collaborations, as it is necessary to regulate the flow of emotions in the group and to avoid potential conflicts.

As shown in Section 3, the notion of coupling/synchrony is not limited to peripheral signals. Brain synchrony has also been related to social processes. Based on this evidence, De Vico et al. (2010) proposed to employ brain connectivity networks, representing both inter-brain and intra-brain synchrony, to predict the decision to defect in an 'iterated prisoner dilemma' game. This game is a two player game where each player can either defect or collaborate with the other player at each round. Connectivity brain networks were constructed from EEG data collected during the decision-making phase of the protocol. These networks were constructed by measuring the partial directed coherence between brain areas of the selfbrain and of the opponent's brain. Finally, a two class multilayer perceptron was trained to predict if both participants would choose to defect in the next round against any other possible strategy. Defection was recognized with an accuracy ranging from 73 to $90 \%$. However, this impressive accuracy is mitigated by the fact that it is obtained based on connectivity networks averaged over several trials. Hence more research is needed to investigate the expected single trial performance.

Physiological coupling is not limited to the analysis of social interactions as it can be also used to evaluate any type of stimulus submitted to several people (Hasson et al., 2012). This can find applications in the field of multimedia tagging, where the common reactions of participants to a movie can indicate highlights or a high interest toward the media. For instance, in Chenes et al. (2013) physiological coupling was used to detect film clip highlights, as reported by participants, with an $F 1$ score of 0.56 , and this despite of the fact that they were not watching the movie together. With a similar approach, the EDA of participants in a real movie audience was employed to create an affective profile of each audience member (Fleureau et al., 2013). These individual affective profiles were then combined to obtain an average affective profile which was found to indicate relevant scenes mentioned by the participants. Concerning central physiological signals, an approach to measure engagement in movie watching from EEG signal correlations was proposed in Dmochowski et al. (2012).

In summary, the few studies employing physiological coupling for prediction have obtained interesting results showing its usefulness for performance assessment and collaborative processes such as emotion management and defection. Furthermore, physiological coupling can also be employed to determine movie highlights and engagement toward media. An aspect of physiological coupling, which was not discussed, is directionality. Directionality defines which of two synchronous systems drives the other. Such information could be used to identify group leaders or dominant persons in a social interaction. Finally, we would also like to stress that physiological coupling might not be the only way to combine physiological signals of several people and research toward finding new approaches is strongly encouraged.

\subsection{Toward applications}

Both individual and group physiological measurements could be employed in several contexts, including mediated collaboration, social entertainment and intimate relationships. The goal of this section is not to review all the potential applications of the proposed measurements but rather to illustrate how physiological signals can be applied to social situations.

Collaboration is an activity which involves the resolution of a common goal by several actors. In this context, providing feedback to individuals about their physiological activity and their emotional state is likely to favor meta-cognition and improve participant's social skills (Jones and Issroff, 2005). This feedback could be achieved in a closed loop where each participant receives only information about its own state and physiology, or disseminated to all the group members following clear ethical rules. Another possibility could be to use individual and group inferences to adapt the interaction. For instance, given that physiological coupling is known to be related to conflicting situations, this measure could be employed to automatically assess the level of conflict in the group. An adaptive computer supported collaborative work environment could then propose solution to help solving conflicts by more focusing the participants' attention to their common objectives and interests.

Entertainment is probably one of the most social activities, people enjoy playing together and they often watch movies in groups. Poker is a nice example of a social game where physiological feedback can play an interesting role, enhancing the player experience (Slovák et al., 2012; Yamabe et al., 2010). On the one hand, it is expected that, by having 
feedback on their own state, the poker players would be able to better control their emotional reactions. On the other hand, giving feedback to the whole group of players informs on who is currently stressed so that they can adapt their playing strategy. These two opposite forms of feedback are expected to create an engaging game experience (Yamabe et al., 2010). Other form of entertainment can also benefit from social physiological measures including cinema. The common physiological responses of spectators can be used to determine the most relevant scene of a movie, for instance for the purpose of movie summarization, to evaluate if the movie elicited the expected emotions, or to create physiological profiles of users for implicit movie tagging and recommendation (Soleymani et al., 2012).

With physiological coupling and feedback being related to empathic accuracy, emotional convergence, perceived connectedness and conflicts, applications can also be found for the management of interpersonal relationships. For instance, empathy detection can be employed to train empathic skills to improve marital stability and decrease marital abuse (Janssen, 2012). These technologies can also be used to maintain connectedness among physically separated members of a family (Slovák et al., 2012). Similarly, physiological coupling could be employed, in the context of a social media website, to recommend connections between people who have similar emotional reactions to movies and potentially similar tastes.

\subsection{Challenges and research directions}

There are more and more devices that can measure physiological signals with very low intrusiveness (Liao et al., 2012) allowing to move toward the applications mentioned in the previous section. Physiological indexes can also be measured by means of cameras either by recording faces (Poh et al., 2010) or by placing your finger on a cell-phone camera (Scully et al., 2012). All these achievements demonstrate the current feasibility of non-intrusive monitoring of physiological signals. However, a challenge specific to multi-person physiological monitoring concerns the synchronization of the different signal sources (i.e. the samples should be time stamped with a common time base). Synchronization is a necessary step to compute physiological coupling and is especially important for high frequency band signals such as EEG. In the laboratory, synchronization can be achieved by sending triggers in parallel to several devices. These triggers generally correspond to specific events of the recording protocol. However, this is not applicable out of the laboratory and, consequently, there is a need to build devices able to synchronize heterogeneous modalities (e.g. different sampling frequencies) automatically.

Physiological signals can be influenced by a multitude of psychological constructs such as emotions, attention and motivation (Ravaja, 2004) but also by any type of physical activity. There is consequently a high risk in the interpretation of physiological responses which are measured in noncontrolled situation (i.e. out of the laboratory). We believe that having information about context is the only solution to solve this problem of confounding variables. For instance, it has been proposed that the observation of differential physiological patterns for the same emotion could be due to differences in context (Stemmler et al., 2001). Similarly, when heart rate information is shared among people they tend to request knowledge about the context to interpret this information (Slovák et al., 2012). Hence, when displaying physiological cues, the context is very important and should not be neglected. For instance, in a remote situation physiological cues should be displayed only with contextual information and when those cues are interpreted (e.g. automatic recognition of affect) context should be integrated in the interpretation.

Similarly to individual physiological measures, physiological coupling measures can be influenced by several sources. At least three such sources have been proposed (Chanel et al., 2012b; Ekman et al., 2012). First physiological coupling can occur due to the perception of common events. For instance, the perception of a strong sound is likely to trigger an attention orientation response in all perceivers (Ravaja, 2004), consequently increasing their physiological coupling. In addition, concordant appraisal of this event among perceivers will increase physiological synchrony further. Secondly, physiological profiles can synchronize because the behaviors and efforts of people are congruent. For instance, it is likely that two people carrying a table together will have to synchronize their actions and efforts, which will lead to an increase in physiological coupling. Thirdly, physiological compliance can emerge from emotional processes such as alignment, convergence, mimicry and emotional contagion (Hatfield et al., 2009; Janssen, 2012). In this case, the physiological profiles of people synchronize because emotions are communicated among the members of the group. There is so far no study which investigated how to differentiate those different sources of physiological synchrony. Hence the aspect of the experimental manipulation, here specifically the coupling source, that is reflected in the changes has to be identified to guarantee valid psychophysiological inferences (Fairclough, 2009) and to avoid spurious coupling measures due to protocol structure (Burgess, 2013).

A first step when employing physiological computing technologies is often to choose which physiological signals will be employed. Most of the studies which investigated physiological feedback have focused on heart beats. This is probably because it is a signal easy to represent (e.g. heart beats sounds or pounding visual hearts) and interpret. There is thus a strong need to study how other signals can be presented to people and to evaluate which features of these signals can enhance social interactions. For instance, as mentioned in Section 4.2, several methods are available to compute physiological coupling (Granger causality, spectrum coherence, phase locking value, etc.). Research is needed to 
investigate the potential relationship existing between them and the types of coupling (i.e. stimuli, behavior or emotional coupling). Another aspect which has not been studied so far is how the dynamic of physiological signals can be interpreted by humans. Studies have focused on the presentation of static physiological cues (e.g. a heartbeat at a given frequency). However, it is known that the dynamic of facial expressions unveils subtle cues about emotions (Bartlett et al., 2014) and hence, it is probable that the dynamic of physiological signals are as well important for their interpretation.

Finally, evaluating the potential accuracy and performance of socio-affective inferences is critical to the development of applications. However, a strongly related research question is how critical the accuracy of such systems actually is. In other words, it is possible that emotion recognition with a low accuracy could be sufficient to satisfy and improve user experience. Although almost every study in physiological computing measures accuracy, only a few have studied its relationship with user experience. In any case, the development of universal datasets is needed to measure the expected accuracy of physiological signals for social-affective assessments. Several datasets have been recorded in the field of SSP (Ěerekoviæ, 2012). However, among the 37 datasets presented in this study only two contain physiological recordings (Koelstra et al., 2012; Soleymani et al., 2012). Furthermore, these two databases are dedicated to emotion recognition in the context of multimedia stimulation and have only little to do with the social situations we are discussing in this paper. To our knowledge, only two studies report on the collection of synchronous physiological dataset (Chanel et al., 2013; Ringeval et al., 2013). Both of them were collected in the context of mediated interactions together with other modalities such as speech, face videos and eye tracking. There is thus a need for new datasets recorded in different contexts, targeting various types of applications, social settings and interaction scenarios. This will allow researchers to compare their results and to investigate the research questions proposed above.

\section{CONCLUSION}

This article describes the research which aims at applying physiological computing to support social interaction. Over the last 20 years, emotion recognition from physiological signals has been more and more studied, even reaching a state where it is close to reach the market. However, researchers in the field generally focused on HCIs, not considering that computers are more and more employed for human-human-mediated communication. In the past decades, research in psychophysiology has studied how social interactions between individuals could be reflected in physiological activity, especially in the amount of its synchronicity. In parallel, the field of social neuroscience has developed to gain insight in the neural structures involved during social interactions. Hyperscanning approaches, consisting of the recording of the joint neurophysiological reactions of two interacting people, have shown that the flow of information communicated between people is reflected in the amount of brain synchrony. Based on the results obtained in neuroscience and social sciences, this article calls for the use of physiological signals to perform social inferences of mediated interactions. First, physiological signals could be displayed, after or before inference, to provide new social cues for mediated interactions. Several social signals, such as facial expressions, already provide information about people's internal states but physiological signals can give cues unavailable with other signals. We believe that such physiological cues could help people to better understand each other, which would enrich current social interactions. Secondly, this article argues for the use of physiological coupling (i.e. a measure of how much the physiological activity of a group of persons is coupled) as a measure of the quality of social interaction and proposes to use this index for direct feedback or for machines adaptation. For instance, being able to assess how strongly people are coupled could be used to drive collaborations toward better performance. Similarly, detecting the directionality of coupling and interdependencies might help to detect leaders in a group. Finally, showing coupling information to a social group might help them to establish social links and to connect with each other. All these potential applications would lead to enhanced social interactions and better connectedness among social groups.

\section{FUNDING}

The first and the last author contributed to this article in equal parts. This research was supported by the National Center of Competence in Research (NCCR) Affective sciences financed by the Swiss National Science Foundation (no. 51NF40104897) and hosted by the University of Geneva.

\section{REFERENCES}

Adolphs, R. (2003) Cognitive neuroscience of human social behaviour. Nat. Rev. Neurosci., 4, 165-178.

Allison, P.D. and Liker, J.K. (1982) Analyzing sequential categorical data on dyadic interaction: a comment on Gottman. Psychol. Bull., 91, 393-403.

Astolfi, L., Toppi, J., De Vico Fallani, F., Vecchiato, G., Salinari, S., Mattia, D., Colosimo, A., Babiloni, F. (2010) Neuroelectrical hyperscanning measures simultaneous brain activity in humans. Brain Topogr., 23, 243-256.

Astolfi, L. et al. (2011a) Study of the Functional Hyperconnectivity Between Couples of Pilots During Flight Simulation: An EEG Hyperscanning Study. In Conference Proceedings: Annual International Conference of the IEEE Engineering in Medicine and Biology Society. IEEE Engineering in Medicine and Biology Society, pp. 2338-2341. IEEE. 
Astolfi, L. et al. (2011b) Imaging the social brain by simultaneous hyperscanning during subject interaction. IEEE Intell. Syst., 26, $38-45$.

Babiloni, F. and Astolfi, L. (2012) Social neuroscience and hyperscanning techniques: past, present and future. Neurosci. Biobehav. Revi. doi:10.1016/j.neubiorev.2012.07.006.

Babiloni, F. et al. (2007a) Cortical Activity and Connectivity of Human Brain During the Prisoner's Dilemma: An EEG Hyperscanning Study. In Conference Proceedings: Annual International Conference of the IEEE Engineering in Medicine and Biology Society. IEEE Engineering in Medicine and Biology Society, pp. 4953-4956.

Babiloni, F. et al. (2007b) High Resolution EEG Hyperscanning During a Card Game. In 29th Annual International Conference of the IEEE Engineering in Medicine and Biology Society, pp. 4957-4960. IEEE.

Babiloni, C. et al. (2012) Brains 'in concert': frontal oscillatory alpha rhythms and empathy in professional musicians. NeuroImage, 60, 105-116.

Barrett, L.F. and Satpute, A.B. (2013) Large-scale brain networks in affective and social neuroscience: towards an integrative functional architecture of the brain. Curr. Opin. Neurobiol., 1-12. doi:10.1016/j.conb.2012.12.012.

Barrett, L.F., Mesquita, B., Ochsner, K.N. and Gross, J.J. (2007) The experience of emotion. Ann. Rev. Psychol., 58, 373-403.

Barry, R.J., Clarke, A.R., Johnstone, S.J., Magee, C.A. and Rushby, J.A. (2007) EEG differences between eyes-closed and eyes-open resting conditions. Clin. Neurophysiol., 118, 27652773.

Barry, R.J., Clarke, A.R., Johnstone, S.J. and Brown, C.R. (2009) EEG differences in children between eyes-closed and eyes-open resting conditions. Clin. Neurophysiol., 120, 1806-1811.

Bartlett, M.S., Littlewort, G.C., Frank, M.G. and Lee, K. (2014) Automatic decoding of facial movements reveals deceptive pain expressions. Current Biol., 24(7), 738-743.

Biocca, F. and Harms, C. (2003a) Guide to the Networked Minds Social Presence Inventory v. 1.2: measures of copresence, social presence, subjective symmetry, and intersubjective symmetry. Retrieved from http://cogprints.org/6743/1/2002_ guide_netminds_measure.pdf.

Biocca, F. and Harms, C. (2003b) Toward a more robust theory and measure of social presence: review and suggested criteria. Presence, 12, 456-480.

Boucsein, W. (1992) Electrodermal Indices of Emotion and Stress. In Electrodermal Activity, pp. 369-391. Springer, New York.

Broek, E.L., van den Schut, M.H., Westerink, J.H.D.M., Herk, J., van Tuinenbreijer, K., van den Broek, E. L. and van Herk, J. (2006) Computing Emotion Awareness through Facial Electromyography. In Huang, T.S., Sebe, N., Lew, M.S., Pavlovic, V., Kölsch, M., Galata, A. and Kisacanin, B. (eds.) ECCV 2006 Workshop on HCI, LNCS 3979, pp. 52-63. Springer, Graz, Austria.

Burgess, A.P. (2013) On the interpretation of synchronization in EEG hyperscanning studies: a cautionary note. Front. Hum. Neurosci., 7,881 .
Cacioppo, J.T., Petty, R.E. and Tassinary, L.G. (1989) Social psychophysiology: a new look. Adv. Exp. Soc. Psychol., 22, 39-91.

Calvo, R.A. and D'Mello, S. (2010) Affect detection: an interdisciplinary review of models, methods, and their applications. IEEE Trans. Affect. Comput., 1, 18-37.

Castellano, G., Villalba, S. and Camurri, A. (2007) Recognising Human Emotions from Body Movement and Gesture Dynamics. In Paiva, A., Prada, R. and Picard, R.W. (eds) Affective Computing and Intelligent Interaction, LNCS 4738, pp. 71-82. Springer, Berlin, Heidelberg.

Castellano, G., Caridakis, G., Camurri, A., Karpouzis, K., Volpe, G. and Kollias, S. (2010) Body Gesture and Facial Expression Analysis for Automatic Affect Recognition. In Scherer, K.R., Baenziger, T. and Roesch, E.B. (eds) Blueprint for Affective Computing: A Sourcebook, pp. 245-255. Oxford University Press, Oxford, England.

Chanel, G., Kierkels, J.J.M., Soleymani, M. and Pun, T. (2009) Shortterm emotion assessment in a recall paradigm. Int. J. Hum.Comput. Stud., 67, 607-627.

Chanel, G., Pelli, S., Ravaja, N. and Kuikkaniemi, K. (2010) Social Interaction Using Mobile Devices and Biofeedback: Effects on Presence, Attraction and Emotions. In BioSPlay Workshop, Fun and Games Conference. Leuven, Belgium. http://www. physiologicalcomputing.net/workshops/biosplay2010/BioSPlay_ Chanel\%20et\%20al\%20\%28Synchro\%29.pdf.

Chanel, G., Rebetez, C., Bétrancourt, M. and Pun, T. (2011) Emotion assessment from physiological signals for adaptation of games difficulty. IEEE Trans. Syst. Man Cybern., Part A, 41, 1052-1063.

Chanel, G., Kalogianni, K., Pun, T. and Betrancourt, M. (2012a) GamEMO: How Physiological Signals Show your Emotions and Enhance your Game Experience. In 14th ACM International Conference on Multimodal Interaction. Santa Monica, CA, USA. ACM.

Chanel, G., Kivikangas, J. M. and Ravaja, N. (2012b) Physiological compliance for social gaming analysis: cooperative versus competitive play. Interact. Comput., 24, 306-3016.

Chanel, G., Molinari, G., Cereghetti, D., Bétrancourt, M. and Pun, T. (2013) Assessment of Computer-Supported Collaborative Processes using Interpersonal Physiological and Eye-Movement Coupling. In Conference on Affective Computing and Intelligent Interaction. Geneva, Switzerland.

Chenes, C., Chanel, G., Soleymani, M. and Pun, T. (2013) Highlights Detection in Movie Scenes Through Inter-Users Physiological Linkage. In Ramzan, N., van Zwol, R., Lee, J.-S., Clüver, K. and Hua, X.-S. (eds) Social Media Retrieval. Springer, London. IEEE.

Coan, J.A. and Allen, J.J.B. (2004) Frontal EEG asymmetry as a moderator and mediator of emotion. Biol. Psychol., 67, 7-50.

Cornelius, R.R. (1996) The Science of Emotion. Research and Tradition in the Psyhcology of Emotion. Prentice-Hall, Upper Saddle River, NJ.

Cowie, R., Douglas-Cowie, E., Tsapatsoulis, N., Votsis, G., Kollias, S., Fellenz, W. and Taylor, J.G. (2001) Emotion recognition in 
human-computer interaction. IEEE Signal Process. Mag., 18, 32-80.

Cui, X., Bryant, D.M. and Reiss, A.L. (2012) NIRS-based hyperscanning reveals increased interpersonal coherence in superior frontal cortex during cooperation. NeuroImage, 59, 2430-2437.

Damasio, A.R. (2001) Fundamental feelings. Nature, 413, 781.

Davidson, R.J. (1995) Cerebral Asymmetry, Emotion, and Affective Style. In Davidson, R.J. and Hugdahl, K. (eds) Brain Asymmetry, pp. 361-387. MIT Press, Cambridge.

De Vico Fallani, F. et al. (2010) Defecting or not defecting: how to 'read' human behavior during cooperative games by EEG measurements. PloS One, 5(12), e14187.

Delaherche, E., Chetouani, M., Mahdhaoui, A., Saint-Georges, C., Viaux, S. and Cohen, D. (2012) Interpersonal synchrony: a survey of evaluation methods across disciplines. IEEE Trans. Affect. Comput., 3, 349-365.

Delplanque, S. et al. (2008) Emotional processing of odors: evidence for a nonlinear relation between pleasantness and familiarity evaluations. Chemical Senses, 33, 469-479.

Dmochowski, J.P., Sajda, P., Dias, J. and Parra, L.C. (2012) Correlated components of ongoing EEG point to emotionally laden attention - a possible marker of engagement? Front. Hum. Neurosci., 6, 112.

Dumas, G. (2011) Towards a two-body neuroscience. Commun. Integrative Biol., 4, 349-352.

Dumas, G., Nadel, J., Soussignan, R., Martinerie, J. and Garnero, L. (2010) Inter-brain synchronization during social interaction. PloS One, 5, 1-10.

Ěerekoviæ, a. (2012) An insight into multimodal databases for social signal processing: acquisition, efforts, and directions. Artif. Intell. Rev. doi:10.1007/s10462-012-9334-2.

Ekman, P., Levenson, R.W. and Friesen, W.V. (1983) Autonomic nervous-system activity distinguishes among emotions. Science, 221, 1208-1210.

Ekman, I., Chanel, G., Kivikangas, J.M., Salminen, M., Järvelä, S. and Ravaja, N. (2012) Social interaction in games: measuring physiological linkage and social presence. Simul. Gaming, 43, 321-338.

Fairclough, S.H. (2009) Fundamentals of physiological computing. Interact. Comput., 21, 133-145.

Féré, C. (1888) Note sur les modifications de la resistance electrique sous l'influence des excitations sensorielles et des emotions. Compt. Rend. Soc. Biol., 5, 217-219.

Fleureau, J., Guillotel, P. and Orlac, I. (2013) Affective Benchmarking of Movies Based on the Physiological Responses of a Real Audience. In 2013 Humaine Association Conference on Affective Computing and Intelligent Interaction, pp. 73-78. IEEE, Geneva, Switzerland.

Frith, C.D. and Frith, U. (2006) How we predict what other people are going to do. Brain Res., 1079, 36-46.

Gendolla, G.H.E. and Richter, M. (2005) Ego involvement and effort: Cardiovascular, electrodermal, and performance effects. Psychophysiology, 42, 596-603.
Gottman, J.M. and Ringland, J.T. (1981) The analysis of dominance and bidirectionality in social-development. Child Dev., 52, 393-412.

Gunes, H., Piccardi, M. and Pantic, M. (2008) From the Lab to the Real World: Affect Recognition Using Multiple Cues and Modalities. In Jimmy, O. (ed.) Affective Computing, Focus on Emotion Expression, Synthesis and Recognition, pp. 185-218. ITech Education and Publishing, Vienna, Austria.

Gunes, H., Shan, C., Chen, S. and Tian, Y. (2013) Bodily Expression for Automatic Affect Recognition. In Konar, A. and Chakraborty, A. (eds) Advances in Emotion Recognition, pp. 1-34. Wiley.

Hareli, S. and Parkinson, B. (2008) What's social about social emotions? J. Theory Soc. Behav., 38, 131-156.

Harmon-Jones, E. (2003) Clarifying the emotive functions of asymmetrical frontal cortical activity. Psychophysiology, 40, 838-848.

Hasson, U., Ghazanfar, A.a., Galantucci, B., Garrod, S. and Keysers, C. (2012) Brain-to-brain coupling: a mechanism for creating and sharing a social world. Trends Cogn. Sci., 1-8. doi:10.1016/j.tics.2011.12.007.

Hatfield, E., Rapson, R.L. and Le, Y.-C.L. (2009) Emotional Contagion and Empathy. In Decety, J. and Ickes, W. (eds) The Social Neuroscience of Empathy, pp. 19-30. MIT Press, Cambridge, Massachusetts.

Hazlett, R.L. and Benedek, J. (2007) Measuring emotional valence to understand the user's experience of software. Int. J. Hum.-Comput. Stud., 65, 306-314.

Henning, R. A., Boucsein, W. and Gil, M. C. (2001). Socialphysiological compliance as a determinant of team performance. Int. J. Psychophysiol., 40, 221-232.

Henning, R. A., Armstead, A. G. and Ferris, J. K. (2009). Social psychophysiological compliance in a four-person research team. Appl. Ergon., 40(6), 1004-1010.

Herff, C., Heger, D., Fortmann, O., Hennrich, J., Putze, F. and Schultz, T. (2013) Mental workload during n-back task-quantified in the prefrontal cortex using fNIRS. Front. Hum. Neurosci., 7, 935.

Hussain, M.S., Alzoubi, O., Calvo, R.A. and D’Mello, S. (2011) Affect Detection from Multichannel Physiology during Learning Sessions with AutoTutor. In Biswas, G., Bull, S., Kay, J. and Mitrovic, A. (eds) Artificial Intelligence in Education, LNCS 6738, pp. 131-138. Springer, Auckland, New Zealand.

Jakobs, E., Fischer, A.H. and Manstead, A.S.R. (1997) Emotional experience as a function of social context: the role of the other. J. Nonverbal Behav., 21, 103-130.

Jalili, M., Barzegaran, E. and Knyazeva, M.G. (2014) Synchronization of EEG: bivariate and multivariate measures. IEEE Trans. Neural Syst. Rehabil. Eng., 22, 212-221.

Janssen, J.H. (2012) A three-component framework for empathic technologies to augment human interaction. J. Multimodal User Interfaces, 6, 143-161.

Janssen, J.H., Bailenson, J.N., IJsselsteijn, W.a. and Westerink, J.H.D.M. (2010) Intimate heartbeats: opportunities for affective 
communication technology. IEEE Trans. Affect. Comput., 1, $72-80$.

Janssen, J.H., Ijsselsteijn, W.A., Westerink, J.H.D.M., Tacken, P. and de Vries, G.-J. (2013a) The tell-tale heart: perceived emotional intensity of heartbeats. Int. J. Synth. Emotions, 4, 65-91.

Janssen, J.H., Tacken, P., de Vries, J.J.G. (Gert-J), van den Broek, E.L., Westerink, J.H.D.M., Haselager, P. and IJsselsteijn, W.a. (2013b) Machines outperform laypersons in recognizing emotions elicited by autobiographical recollection. Hum.-Comput. Interact., 28, 479-517.

Jensen, O. and Tesche, C.D. (2002) Frontal theta activity in humans increases with memory load in a working memory task. Eur. J. Neurosci., 15, 1395-1399.

Jiang, J., Dai, B., Peng, D., Zhu, C., Liu, L. and Lu, C. (2012) Neural synchronization during face-to-face communication. J. Neurosci., 32, 16064-16069.

Jones, A. and Issroff, K. (2005) Learning technologies: affective and social issues in computer-supported collaborative learning. Comput. Educ., 44, 395-408.

Katsis, C.D., Katertsidis, N., Ganiatras, G. and Fotiadis, D.I. (2008) Toward emotion recognition in car racing drivers: a biosignal processing approach. IEEE Trans. Syst. Man Cybernet.-Part A: Syst. Hum., 38, 502-512.

Kilner, J.M. and Frith, C.D. (2008) Action observation: inferring intentions without mirror neurons. Current Biol., 18(1), R32-R33.

Kivikangas, J.M., Chanel, G., Cowley, B., Ekman, I., Salminen, M., Järvelä, S. and Ravaja, N. (2011) A review of the use of psychophysiological methods in game research. J. Gaming Virtual Worlds, 3, 181-199.

Kleinsmith, A., Bianchi-Berthouze, N. and Steed, A. (2011) Automatic recognition of non-acted affective postures. IEEE Trans. Syst. Man Cybernet., Part B: Cybernet., PP, 1-12.

Klimesch, W. (1996) Memory processes, brain oscillations and EEG synchronization. Int. J. Psychophysiol., 24, 61-100.

Koelstra, S. and Patras, I. (2013) Fusion of Facial expressions and EEG for implicit affective tagging. Image Vision Comput., 31, 164-174.

Koelstra, S. et al. (2012) DEAP: a database for emotion analysis using physiological signals. IEEE Trans. Affect. Comput., 3, 18-31.

Konvalinka, I., Bauer, M., Stahlhut, C., Hansen, L.K., Roepstorff, A. and Frith, C. D. (2014) Frontal alpha oscillations distinguish leaders from followers: multivariate decoding of mutually interacting brains. NeuroImage, 94, 79-88.

Kosunen, I., Kuikkaniemi, K., Laitinen, T. and Turpeinen, M. (2010) Demonstration: Listen to Yourself and Others-Multiuser Mobile Biosignal Sonification Platform EMOListen. In BioSPlay Workshop, Fun and Games Conference. Leuven, Belgium. http://www.physiologicalcomputing.net/workshops/biosplay2010/ BioSPLay_Kosunen\%20et\%20al\%20\%28Emolisten\%29.pdf.

Kreibig, S.D. (2010) Autonomic nervous system activity in emotion: a review. Biol. Psychol., 84, 394-421.
Lang, P.J., Greenwald, M.K., Bradley, M.M. and Hamm, A.O. (1993) Looking at pictures: affective, facial, visceral, and behavioral reactions. Psychophysiology, 30, 261-273.

Laursen, B., Jensen, B.R., Garde, A.H. and Jørgensen, A.H. (2002) Effect of mental and physical demands on muscular activity during the use of a computer mouse and a keyboard. Scand. J. Work Environ. Health, 28, 215-221.

Le Van Quyen, M., Foucher, J., Lachaux, J., Rodriguez, E., Lutz, A., Martinerie, J. and Varela, F. J. (2001) Comparison of Hilbert transform and wavelet methods for the analysis of neuronal synchrony. J. Neurosci. Methods, 111, 83-98.

Lehmann-Willenbrock, N., Meyers, R.a., Kauffeld, S., Neininger, a. and Henschel, a. (2011) Verbal interaction sequences and group mood: exploring the role of team planning communication. Small Group Res., 42, 639-668.

Levenson, R.W. and Gottman, J.M. (1983) Marital interaction: physiological linkage and affective exchange. J. Pers. Soc. Psychol., 45, 587-597.

Levenson, R.W. and Ruef, A.M. (1992) Empathy: a physiological substrate. J. Pers. Soc. Psychol., 63, 234-246.

Liao, L.-D. et al. (2012) Biosensor technologies for augmented brain-computer interfaces in the next decades. Proc. IEEE, 100, 1553-1566.

Lindenberger, U., Li, S.C., Gruber, W. and Muller, V. (2009) Brains swinging in concert: cortical phase synchronization while playing guitar. BMC Neurosci., 10, 22.

Lindquist, K.a and Barrett, L.F. (2012) A functional architecture of the human brain: emerging insights from the science of emotion. Trends Cogn. Sci., 16, 533-540.

Lisetti, C.L. and Nasoz, F. (2004) Using noninvasive wearable computers to recognize human emotions from physiological signals. J. Appl. Signal Process., 11, 1672-1687.

Liu, C., Agrawal, P., Sarkar, N. and Chen, S. (2009) Dynamic difficulty adjustment in computer games through real-time anxiety-based affective feedback. Int. J. Hum.-Comput. Interact., 25, 506-529.

Mandryk, R.L., Inkpen, K.M. and Calvert, T.W. (2006) Using psychophysiological techniques to measure user experience with entertainment technologies. Behav. Inf. Technol., 25, $141-158$.

Mehu, M. and Scherer, K.R. (2012) A psycho-ethological approach to social signal processing. Cogn. Process., 13, 397-414.

Ménoret, M. et al. (2014) Neural correlates of non-verbal social interactions: a dual-EEG study. Neuropsychologia, 55, 85-97.

Molenberghs, P., Cunnington, R. and Mattingley, J.B. (2009) Is the mirror neuron system involved in imitation? A short review and meta-analysis. Neurosci. Biobehav. Rev., 33, 975-980.

Mühl, C. et al. (2010) Bacteria hunt-evaluating multi-paradigm BCI interaction. J. Multimodal User Interfaces, 4, 11-25.

Mühl, C., Brouwer, A.-M., van Wouwe, N., van den Broek, E.L., Nijboer, F. and Heylen, D.K.J. (2011) Modality-Specific Affective 
Responses and their Implications for Affective BCI. In MüllerPutz, G.R., Scherer, R., Billinger, M., Kreilinger, A., Kaiser, V. and Neuper, C. (eds) Proceedings of the Fifth International BrainComputer Interface Conference 2011, Graz, Austria. Verlag der Technischen Universität, Graz, Austria.

Mühl, C., Allison, B., Nijholt, A. and Chanel, G. (2014a) A survey of affective brain computer interfaces: principles, state-of-the-art, and challenges. Brain-Comput. Interfaces, 1, 66-84.

Mühl, C., Jeunet, C. and Lotte, F. (2014b) EEG-based workload estimation across affective contexts. Front. Neurosci., 8, 114.

Müller, V., Sänger, J. and Lindenberger, U. (2013) Intra- and interbrain synchronization during musical improvisation on the guitar. PloS One, 8, e73852.

Naeem, M., Prasad, G., Watson, D.R. and Kelso, J.a.S. (2012) Electrophysiological signatures of intentional social coordination in the 10-12 Hz range. NeuroImage, 59, 1795-1803.

Niedermeyer, E. (2005) The Normal EEG of the Waking Adult. In Niedermeyer, E. and Lopes Da Silva, F. (eds) Electroencephalography Basic Principles Clinical Applications and Related Fields, pp. 167-192. Williams \& Wilkins.

Novak, D., Mihelj, M. and Munih, M. (2012) A survey of methods for data fusion and system adaptation using autonomic nervous system responses in physiological computing. Interact. Comput., 24, 154-172.

Ortony, A., Revelle, W. and Zinbarg, R. (2007) Why Emotional Intelligence Needs a Fluid Component. In Matthews, G., Zeidner, M. and Roberts, R.D. (eds) The Science of Emotional Intelligence, pp. 288-304. Oxford University Press.

Pantic, M. and Rothkrantz, L.J.M. (2003) Toward an affectsensitive multimodal human-computer interaction. Proc. IEEE, 91, 1370-1390.

Pentland, A.S. (2006) Collective intelligence. August, 9-12.

Pentland, A.S. (2007) Social signal processing. IEEE Signal Process. Mag., July, 108-111.

Pesarin, A., Cristani, M., Murino, V. and Vinciarelli, A. (2012). Conversation analysis at work: detection of conflict in competitive discussions through semi-automatic turn-organization analysis. Cogn. Process., 13, 533-540.

Pfurtscheller, G., Stancák, A. and Neuper, C. (1996) Event-related synchronization (ERS) in the alpha band-an electrophysiological correlate of cortical idling: a review. Int. J. Psychophysiol., 24, $39-46$.

Picard, R.W. (1997) Affective computing. The MIT Press.

Poh, M.-Z., McDuff, D.J. and Picard, R.W. (2010) Non-contact, automated cardiac pulse measurements using video imaging and blind source separation. Optics Express, 18, 1076210774.

Rainville, P., Bechara, A., Naqvi, N. and Damasio, A.R. (2006) Basic emotions are associated with distinct patterns of cardiorespiratory activity. Int. J. Psychophysiol., 61, 5-18.

Ravaja, N. (2004) Contributions of phsychophysiology to media research: review and recommendations. Media Psychol., 6, 193235
Ringeval, F., Sonderegger, A., Sauer, J. and Lalanne, D. (2013) Introducing the RECOLA Multimodal Corpus of Remote Collaborative and Affective Interactions. In 10th IEEE International Conference and Workshops on Automatic Face and Gesture Recognition (FG), pp. 1-8. IEEE.

Rizzolatti, G. and Craighero, L. (2004) The mirror-neuron system. Ann. Rev. Neurosci., 27, 169-192.

Rizzolatti, G. and Fabbri-Destro, M. (2008) The mirror system and its role in social cognition. Curr. Opin. Neurobiol., 18, 179-184.

Sallnäs, E.-L. (2005) Effects of communication mode on social presence, virtual presence, and performance in collaborative virtual environments. Presence, 14, 434-449.

Sanchez-Cortes, D., Aran, O., Babu Jayagopi, D., Schmid Mast, M. and Gatica-Perez, D. (2012) Emergent leaders through looking and speaking: from audio-visual data to multimodal recognition. J. Multimodal User Interfaces, August.

Sander, D., Grandjean, D. and Scherer, K.R. (2005) A systems approach to appraisal mechanisms in emotion. Neural Netw., 18, 317-352.

Sänger, J., Lindenberger, U. and Müller, V. (2011) Interactive brains, social minds. System, December, 655-663.

Sassaroli, A., Zheng, F., Hirshfield, L.M., Girouard, A., Solovey, E.T., Jacob, R.J.K., and Fantini, S. (2008) Discrimination of mental workload levels in human subjects with functional near-infrared spectroscopy. J. Innov. Optical Health Sci., 01, 227-237.

Schilbach, L., Timmermans, B., Reddy, V., Costall, A., Bente, G., Schlicht, T. and Vogeley, K. (2013) Toward a second-person neuroscience. Behav. Brain Sci., 36, 393-414.

Schmidt, L.A. and Trainor, L.J. (2001) Frontal brain electrical activity (EEG) distinguishes valence and intensity of musical emotions. Cogn. Emotion, 15, 487-500.

Scully, C.G., Lee, J., Meyer, J., Gorbach, A.M., Granquist-Fraser, D., Mendelson, Y. and Chon, K.H. (2012) Physiological parameter monitoring from optical recordings with a mobile phone. IEEE Trans. Biomed. Eng., 59, 303-306.

Slovák, P., Janssen, J. and Fitzpatrick, G. (2012) Understanding Heart Rate Sharing: Towards Unpacking Physiological Space. In Proceedings of the 2012 ACM annual conference on Human Factors in Computing Systems-CHI'12, p. 859. ACM Press, New York, New York, USA.

Soleymani, M., Lichtenauer, J., Pun, T. and Pantic, M. (2012) A multimodal database for affect recognition and implicit tagging. IEEE Trans. Affect. Comput., 3, 42-55.

Stemmler, G., Heldmann, M., Pauls, C.A. and Scherer, T. (2001) Constraints for emotion specificity in fear and anger: the context counts. Psychophysiology, 38, 275-291.

Tangney, J.P., Stuewig, J. and Mashek, D.J. (2007) Moral emotions and moral behavior. Ann. Rev. Psychol., 58, 345-372.

Tognoli, E., Lagarde, J., DeGuzman, G.C. and Kelso, J.a.S. (2007) The phi complex as a neuromarker of human social coordination. Proc. Natl Acad. Sci. USA, 104, 8190-8195. 
Treder, M.S., Bahramisharif, A., Schmidt, N.M., van Gerven, M.A.J. and Blankertz, B. (2011) Brain-computer interfacing using modulations of alpha activity induced by covert shifts of attention. J. Neuroeng. Rehabil., 8, 24.

Uddin, L.Q., Iacoboni, M., Lange, C. and Keenan, J. P. (2007) The self and social cognition: the role of cortical midline structures and mirror neurons. Trends Cogn. Sci., 11(4), 153-157.

Van Kleef, G.a. (2009) How Emotions Regulate Social Life. Curr. Dir. Psychol. Sci., 18, 184-188.

Van Kleef, G. a., De Dreu, C. K. W., Pietroni, D. and Manstead, A. S. R. (2006). Power and emotion in negotiation: power moderates the interpersonal effects of anger and happiness on concession making. Eur. J. Soc. Psychol., 36, 557-581.

Verwey, W.B. and Veltman, H.A. (1984) Detecting short periods of elevated workload: a comparison of nine workload assessment techniques. J. Exp. Psychol. Appl., 2, 270-285.

Vinciarelli, A., Pantic, M. and Bourlard, H. (2009) Social signal processing: Survey of an emerging domain. Image Vision Comput., 27, 1743-1759.

Vinciarelli, A., Pantic, M., Heylen, D., Pelachaud, C., Poggi, I., D'Errico, F. and Schroeder, M. (2012) Bridging the gap between social animal and unsocial machine: a survey of social signal processing. IEEE Trans. Affect. Comput., 3, 69-87.

Vytal, K. and Hamann, S. (2010) Neuroimaging support for discrete neural correlates of basic emotions: a voxel-based meta-analysis. J. Cogn. Neurosci., 22, 2864-2885.

Wilhelm, F.H., Pfaltz, M.C. and Grossman, P. (2006) Continuous electronic data capture of physiology, behavior and experience in real life: towards ecological momentary assessment of emotion. Interact. Comput., 18, 171-186.

Yamabe, T., Kosunen, I., Ekman, I., Liikkanen, L.A., Kuikkaniemi, K. and Nakajima, T. (2010) Biofeedback Training with EmoPoker: Controlling Emotional Arousal for Better Poker Play. In BioSPlay Workshop, Fun and Games Conference. Leuven, Belgium.

Yun, K., Chung, D. and Jeong, J. (2008) Emotional Interactions in Human Decision-Making using EEG Hyperscanning. Proceedings of the 6th International Conference on Cognitive Science, pp. 327-330. http://cogsci.snu.ac.kr/jcs/iccs/.

Zeng, Z.H., Pantic, M., Roisman, G.I. and Huang, T.S. (2009) A survey of affect recognition methods: audio, visual, and spontaneous expressions. IEEE Trans. Pattern Anal. Mach. Intell., $31,39-58$. 